\title{
Regenerative and Antioxidant Properties of Autologous Platelet- Rich Plasma Can Reserve the Aging Process of the Cornea in the Rat Model
}

\author{
Heba R. Hashem \\ Anatomy and Embryology Department, Faculty of Medicine, Ain Shams University, Cairo, Egypt \\ Correspondence should be addressed to Heba R. Hashem; hebahramadan@gmail.com
}

Received 12 June 2020; Revised 14 September 2020; Accepted 7 November 2020; Published 23 November 2020

Academic Editor: Marina Sokovi

Copyright (C) 2020 Heba R. Hashem. This is an open access article distributed under the Creative Commons Attribution License, which permits unrestricted use, distribution, and reproduction in any medium, provided the original work is properly cited.

\begin{abstract}
Aging is a natural progressive decline in the biological function of cells. Age-related changes in the cornea can affect its ability to refract light or repair itself. Platelet-rich plasma (PRP) has a promising role in regenerative medicine and evidenced its efficacy in multiple fields, but in corneal aging has not yet been elucidated. The present work was performed to estimate the regenerative antioxidant effect of PRP on corneal aging in rats. Rats were assigned into two main groups: (GI) adult group and (GII) aged group. The adult group was divided into GIa (adult rats), GIb (adult-saline treated), and GIc (adult-PRP treated). The aged group was divided into GIIa (aged rats) and GIIb (aged, PRP treated). PRP was administered by a single subconjunctival injection. After 10 days, histological, ultrastructural, immunohistochemical, and morphometrical investigations were carried out. Examination of the corneal sections of the aged group revealed corneal epithelial thinning, shedding of the surface epithelium with loss of desmosomal junction, and irregularity in Bowman's membrane. Disorganized widely spaced collagen bundles and neovascularization were detected in corneal stroma associated with thickening in Descemet's membrane. Ultrastructural examination revealed shrunken hyperchromatic nuclei, swollen mitochondria, and scanty cytoplasm with a strong nuclear reaction for caspase-3 immunostaining. Moreover, antioxidant/free radicals' imbalance was detected by the increase of malondialdehyde (MDA) level with a decrease of glutathione peroxidase (GPx) and superoxide dismutase (SOD) levels. In contrast, GIIb (aged, PRP treated) section examination revealed a restoration of the thickness of the corneal epithelial layer and Descemet's membrane with an amendment of collagen fiber regularity that is associated with weak nuclear reaction to caspase- 3 and recovery of the balance in the redox state. These findings proved the effectiveness of PRP as a promising regenerative treatment for the age-associated changes in the cornea.
\end{abstract}

\section{Introduction}

The cornea is the transparent anterior part of the eye that covers the iris, pupil, and the anterior chamber. Its major functions are to support the tear film and surface refractivity and to transmit light through its translucent tissue to the lens and then to the retina [1]. Corneal hydration is vital to protect the corneal epithelium from injuries and preservation of corneal transparency [2].

The cornea is formed of five distinct histological layers arranged from outer to inner, respectively, corneal epithelium, Bowman's membrane, stroma, Descemet's membrane, and endothelium.
The corneal epithelium (stratified squamous nonkeratinized) is one of the most sensitive and densely innervated surface tissues in the human body. It promotes the maintenance of reflex tear production and the physiologic renewal of the corneal epithelium [3]. Corneal stroma represents almost $90 \%$ of the corneal thickness [4].

The integrity of the stroma and the normal metabolism of endothelial cells are important factors to preserve corneal transparency [2]. The transparency depends on the regular spacing and uniform diameter of the collagen bundles in the stroma [5].

Moreover, Descemet's membrane is the basement membrane of the corneal endothelium cell layer. The endothelial 
cell layer provides a barrier function and acts as an active water pump to keep the cornea in a constant state of dehydration [6]. People are born with a fixed number of corneal endothelial cells that gradually decreased with age [1].

Aging is a natural biological progressive process characterized by a decrease in cellular and molecular tissue functions [7]. Corneal aging produces both structural and functional changes that have a major effect on vision [8]. These changes may include keratoconus, alterations of higher order aberrations of the cornea, and a rotation of the axis of astigmatism resulting in a shift from with-therule to against-the-rule astigmatism [9]. Age-related alterations lead to dry eye, which in turn leads to a decrease in visual acuity, discomfort, subjacent epithelial injury, and inflammation [1].

Platelet-rich plasma (PRP) becomes an attractive way of treatment in several fields like regenerative medicine, dental and plastic surgical applications, ophthalmological surgery, trauma, and skin burns [10].

PRP is an endogenously derived therapeutic technology so it is a nontoxic and nonimmunogenic technique used to accelerate and stimulate tissue healing [11]. PRP is obtained from centrifugation for the blood sample to get cellular constitute with a platelet concentration higher than that in circulating blood [12].

PRP was found to promote tissue regeneration by enhancing cell proliferation and differentiation [13]. Platelets secrete several growth factors, including insulin-like growth factors, platelet-derived growth factor, vascular endothelial growth factor, and fibroblast growth factor [11, 14]. These growth factors promote the process of collagen formation, angiogenesis, and regeneration. Moreover, the antimicrobial ability of PRP was proved by leukocytes' presence that lowering the risk of infection [13].

The present study is conducted to assess the efficacy of autologous platelet-rich plasma on the histopathological changes that occurred in the cornea during the aging process.

\section{Materials and Methods}

2.1. Animals. Thirty male albino rats of Wistar strain were used in the present study. Eighteen of the rats were aged 36 months old and were considered as adult rats, and the rest were aged 22-26 months old and were considered as aged rats [15]. Animals were obtained from the animal house of Research Center and Bilharzial Research Unit of Faculty of Medicine, Ain Shams University.

Rats were allowed free access to water and food and were housed in a wire cage with 12 hours day and night cycle. The animals were kept in adjusted laboratory conditions (temperature $21 \pm 3^{\circ} \mathrm{C}$, well-ventilated wire cages). Animals were left one week for acclimatization before the start of the experiment.

2.2. Ethical Consideration. All the experiments and animal procedures were conducted following the national guidelines approved by the Committee of Animal Research Ethics (CARE), Faculty of Medicine, Ain Shams University, and fol- lowing the NIH Guidelines for the Care and Use of Laboratory Animals 8th edition.

2.3. Experimental Design. In Group I, twelve adult rats were divided randomly into two groups as follows:

Ia (adult, untreated group): six adult rats were not subjected to any procedure

Ib (adult, saline-treated group): six adult rats received a single subconjunctival injection of $0.5 \mathrm{ml}$ of saline then left untreated for the rest of the experiment (10 days)

Ic (adult, PRP treated group): six adult rats received a single subconjunctival injection of $0.5 \mathrm{ml}$ of platelet-rich plasma (PRP) then were left untreated for the rest of the experiment (10 days) [14]

In Group II, twelve aged rats were divided randomly into two groups as follows:

IIa (aged, untreated group): six aged rats were not subjected to any procedure

IIb (aged, PRP-treated group): six aged rats received a single subconjunctival injection of $0.5 \mathrm{ml}$ of platelet-rich plasma (PRP) then were left untreated for the rest of the experiment (10 days) [14]

2.4. Preparation of Platelet-Rich Plasma (PRP). Blood samples were collected from the rats to be injected by their PRP. Rats were anesthetized by intraperitoneal injection of $10 \%$ chloral hydrate $(350 \mathrm{mg} / \mathrm{kg}$ body weight) [16]. Venous blood was collected from the tail vein in acid citrate dextrose (ACD) tubes. The samples were centrifuged at $1480 \mathrm{rpm}$ for 6 minutes at $20^{\circ} \mathrm{C}$ to sediment down red blood cells (RBCs). The supernatant plasma containing platelets was transferred into another sterile tube without anticoagulant. Secondly, the centrifuge was done at $4000 \mathrm{rpm}$ for 15 minutes at $20^{\circ} \mathrm{C}$ to separate platelet-rich plasma (PRP) (lower 1/3rd) from platelet-poor plasma (PPP) (the upper 2/3rd) [17]. Samples were centrifuged by using Jouan ki22 Refrigerated Centrifuge (LAB EQUIP LTD, France) and processed at the Tissue Culture and Research Center in the Faculty of Medicine, Al-Azhar University, Egypt.

At the end of the experiment, all rats were euthanized by an intraperitoneal injection of phenobarbital $(50 \mathrm{mg} / \mathrm{kg}$ body weight) [18]. Then, the eyes were enucleated. Some were fixed in $10 \%$ formal saline; the cornea was dissected and processed for light microscopy. Others were fixed in glutaraldehyde and processed for the ultrastructural study.

2.5. Light Microscopic Study. Specimens were fixed in $10 \%$ neutral-buffered formalin, dehydrated in graded alcohol, cleared in xylol, and embedded in paraffin. Sections of $5 \mu \mathrm{m}$ thickness were stained with.

(1) Hematoxylin and Eosin (HE) staining method [19]

(2) Masson trichrome staining method: for the detection of collagen fibers [19]

The slides were examined and photographed with the Lecia ICC50 camera. 
2.6. Immunohistochemical Study. Five $\mu \mathrm{m}$ thick corneal sections were obtained from paraffin blocks, and the slides were dried and deparaffinized. Slides were washed in PBS. An antigen retrieval solution was applied for 10 minutes and endogenous incubation in $3 \% \mathrm{H} 2 \mathrm{O} 2$ for 10 minutes. The sections were incubated with rabbit polyclonal anticaspase-3 (Abcam, ab4051; Cambridge, UK, dilution $1: 100$ ) at room temperature for 90 minutes. Sections were then washed several times with PBS then incubated with secondary antibody (catalog number ab205718, Abcam, Cambridge, UK) at room temperature for 20 minutes [20]. Negative control sections were performed with the same procedure, but the primary antibody was nonimmune rabbit serum.

2.7. Ultrastructural Study. Corneal specimens from sacrificed rats were obtained and fixed in glutaraldehyde and osmium tetroxide. The fixed parts were dehydrated and embedded in Epon resin. Semithin sections, $1 \mu \mathrm{m}$ thick was cut and stained with $1 \%$ toluidine blue and examined by a light microscope to choose the selected areas for proper orientation. Ultrathin sections $(80-90 \mathrm{~nm})$ were cut with a diamond knife and stained by Uranyl acetate and lead citrate [21].

The electron microscopic study was performed with a Jeol 1010 Transmission Electron Microscope (Japan) at the Regional Center for Mycology and Biotechnology, Al-Azhar University, Egypt.

2.8. Tissue Homogenate (Determination of Redox Status in the Cornea). The tissue samples were homogenized in cold phosphate buffered saline (PBS; $10 \% w / v)$ using a tissue homogenizer. The homogenate was centrifuged at 10,000 rpm for $20 \mathrm{~min}$ at $4^{\circ} \mathrm{C}$, and the supernatant was collected.

2.8.1. Malondialdehyde (MDA) Level. It is the indicator of lipid peroxidation (Colorimetric/Fluorometric Assay Kit, Catalog \# K739-100, BioVision, USA). $10 \mathrm{mg}$ of the sample tissue was blended with $150 \mu \mathrm{l} \mathrm{dH} 2 \mathrm{O}, 3 \mu \mathrm{l} \mathrm{BHT}$, and 1 vol of $2 \mathrm{~N}$ perchloric acid, vortexing then centrifuged to remove precipitated protein for $10 \mathrm{~min}(4000 \mathrm{rpm})$.

2.8.2. Glutathione Peroxidase Activity (GPx). Glutathione peroxidase family of enzymes plays an important role in the protection from oxidative damage (Colorimetric Assay Kit, Catalog \#K762-100; 100 reactions; Store kit at $\left.-20^{\circ} \mathrm{C}\right) .0 .1 \mathrm{~g}$ tissue sample was homogenized then centrifuge for $15 \mathrm{~min}$.

2.8.3. Superoxide Dismutase (SOD). It is a group of enzymes that catalyze the superoxide radicals to hydrogen peroxide thus providing cellular defense against reactive oxygen species (Colorimetric Assay Kit, Catalog \#K335-100; Store kit at $\left.-4^{\circ} \mathrm{C}\right)$. Tissue samples were washed by PBS then centrifuged at $500 \mathrm{rpm}$ for $5 \mathrm{~min}$.

All tissue homogenate samples were processed at Tissue Culture and Research Center, Histology Department, AlAzhar University.

2.9. Morphometric Study. Images were analyzed using computer-based software, the image analyzer Leica (Q 500 MC program, Wetzlar, Germany) at Histology Department, Faculty of Medicine, Al-Azhar University, Egypt. Six differ- ent nonoverlapping randomly selected fields obtained from different animals from the same group were used to measure:

(1) The thickness of the corneal epithelium in $\mathrm{HE}$ sections

(2) The thickness of Descemet's membrane in $\mathrm{HE}$ sections

(3) Area of the percentage of caspase-3 positive cells in immunohistochemically stained sections

2.10. Statistical Analysis. The obtained data were statistically analyzed using the SPSS statistical package (IBM Corporation, New York, USA), and data are presented as mean \pm standard deviation (SD). Statistical analysis was performed using one-way analysis of variance (ANOVA) followed by a Tukey's post hoc multiple comparison test. The difference was considered significant at $\mathrm{p}<0.05$ and highly statistically significant at $p<0.001$.

\section{Results}

Light and electron microscopic examination of sections of the cornea from groups Ia, Ib, and Ic showed similar findings with no observable differences. Thus, they were represented as the adult group (I) in figures.

3.1. Histological Results. Examination of HE stained sections of the cornea obtained from the adult group showed the typical structure of the cornea; the corneal epithelium is the outer layer which was formed of stratified squamous nonkeratinized epithelium appeared with superficial flat squamous cells having flat nuclei, intermediate polygonal cells with rounded nuclei, and basal columnar cells with oval nuclei. The second layer is the Bowman's membrane which was a thin homogenous layer consisting of fibrous tissue that lies beneath the corneal epithelium (Figures 1(a) and 1(b)). The avascular corneal stroma was the third layer. It contains regularly arranged bundles of collagen fibers with spindleshaped keratocytes in between (Figures 1(a)-1(c)). The next layer the Descemet's membrane which appeared homogenous continuous beneath the stroma and was covered by an inner single layer of flat endothelial cells which was the fifth layer (Figures 1(a) and 1(c)).

Examination of HE-stained sections of the aged group showed disfigurement of the normal corneal histological structure. The corneal epithelial layer showed focal discontinuity and denudation in the surface epithelium. The basal and intermediate layer appeared with pale vacuolated cytoplasm and deeply stained nuclei. Bowman's membrane appeared very thin, irregular with areas of disruption (Figures 1(d) and 1(e)). Stromal inflammatory cellular infiltration and neovascularization were observed accompanied by disruption, irregularly arranged, and widely spacing collagen bundles (Figures 1(d) and 1(f)). Descemet's membrane appeared irregular and degenerated with areas of separation from the corneal stroma. That was accompanied by an irregular arrangement or loss of some endothelial cells (Figure 1(f)). 


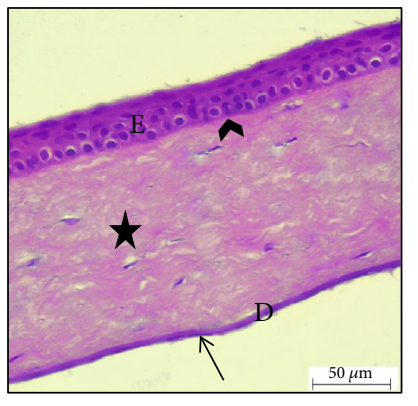

(a)

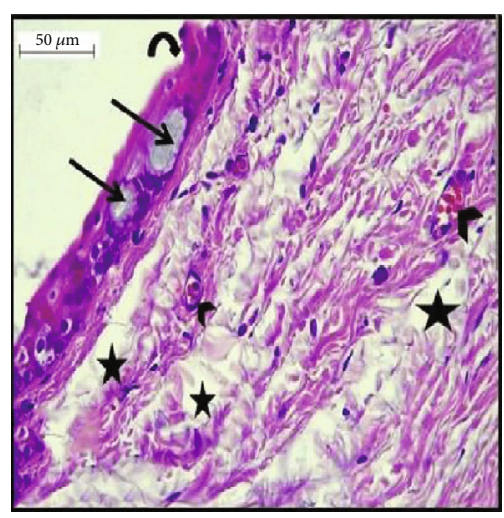

(d)

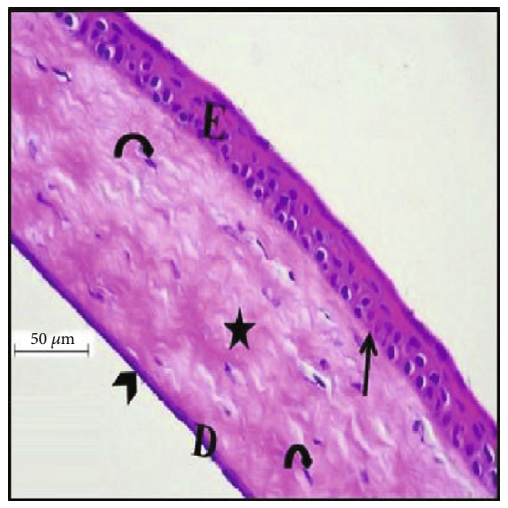

(g)

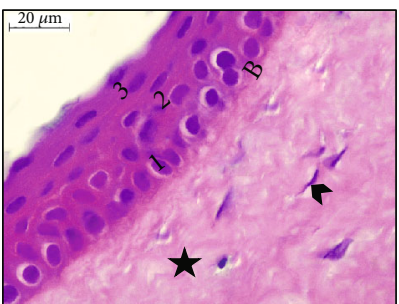

(b)

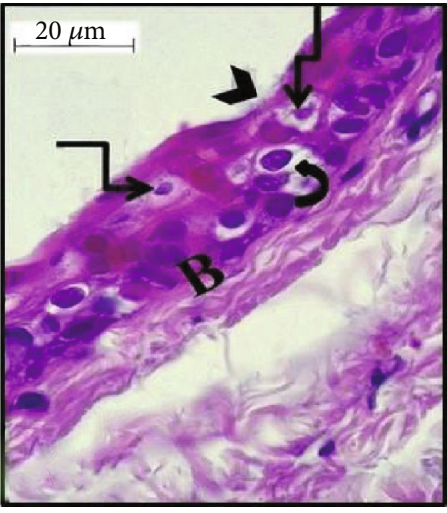

(e)

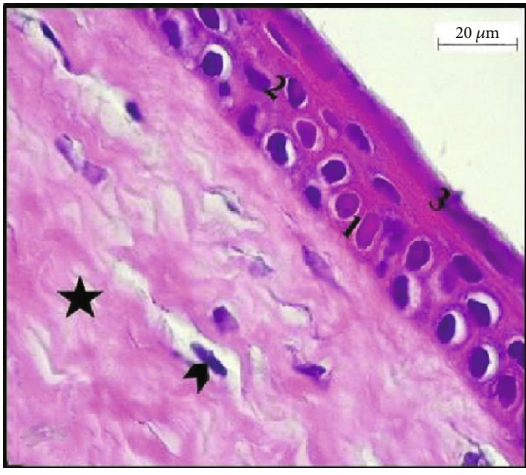

(h)

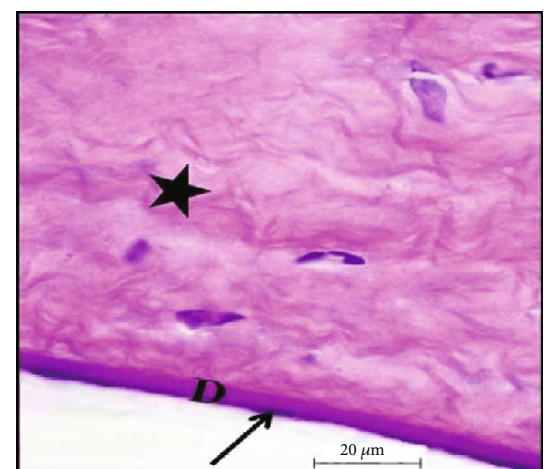

(c)

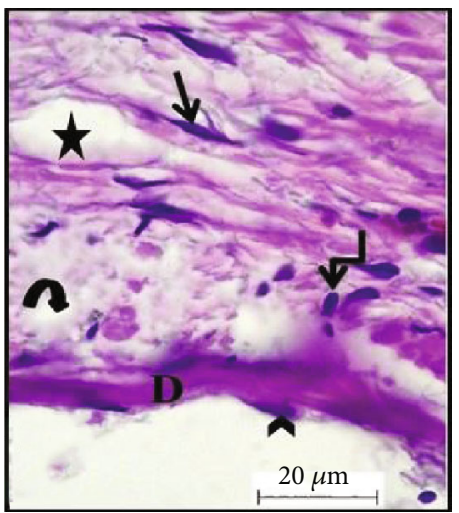

(f)

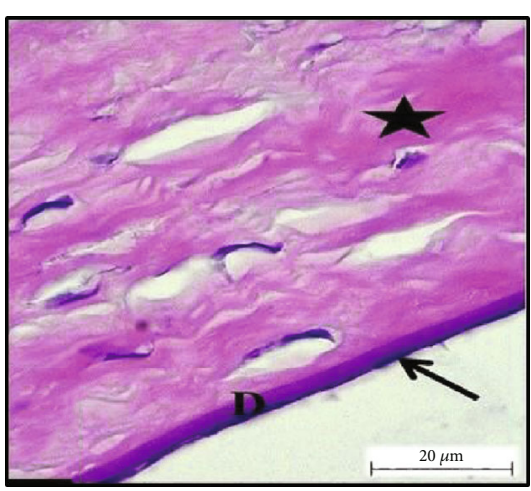

(i)

FIGURE 1: Photomicrographs of HE sections in the rat cornea showing. Group I (adult): (a) the five layers of the cornea: the corneal epithelium (E), Bowman's membrane (arrowhead), corneal stroma (asterisk), and Descemet's membrane (D). Notice the flat endothelial cells (arrow) (HE $\times 400)$; (b) Bowman's membrane (B) and layers of the corneal epithelium: basal layer (1), the intermediate layer (2), and the superficial layer (3). Notice the corneal stroma with bundles of collagen fibers (asterisk) and keratocytes in between (arrowhead) (HE $\times 1000$ ); (c) collagen bundles (asterisk), Descemet's membrane layer (D), and a single layer of flat endothelial cells (arrow) on its inner surface $(\mathrm{HE} \times 1000)$. Group IIa (aged): (d) focal discontinuity in the surface epithelium (curved arrow), pale vacuolated degenerated basal, and intermediate cells (arrow). Notice the neovascularization (arrowhead) and irregularly arranged widely spaced collagen bundles (asterisk) in the stroma ( $\mathrm{HE} \times 400)$; (e) corneal epithelial layers with focal discontinuity in the surface epithelium (arrowhead) with shrunken nuclei (angular arrow) and vacuolated cytoplasm (curved arrow). Notice the thin irregular Bowman's membrane with an area of focal disruption (B) $(\mathrm{HE} \times 1000)$; (f) stromal inflammatory cellular infiltration (angular arrow), keratocytes (arrow), and irregularly arranged widely spaced collagen bundles (asterisk). Notice the irregular degenerated Descemet's membrane (D) with areas of separation from corneal stroma (curved arrow) and irregularly arranged endothelial cells (arrowhead) (HE $\times 1000)$. Group IIb (aged, PRP treated): (g) the cornea layers arranged in its normal order as corneal epithelium (E), Bowman's membrane (arrow), stromal collagen fibers (asterisk) with keratocytes (curved arrow), and Descemet's membrane (D) with endothelial cells (arrowhead) (HE $\times 400)$; (h) layers of the corneal epithelium: basal layer (1), the intermediate layer (2), the superficial layer (3), and the stroma collagen fibers (asterisk) with keratocytes in between (arrowhead) (HE $\times 1000)$; (i) Descemet's membrane layer (D) and a single layer of flat endothelial cells (arrow) on its inner surface. Notice collagen bundles (asterisk) $(\mathrm{HE} \times 1000)$. 


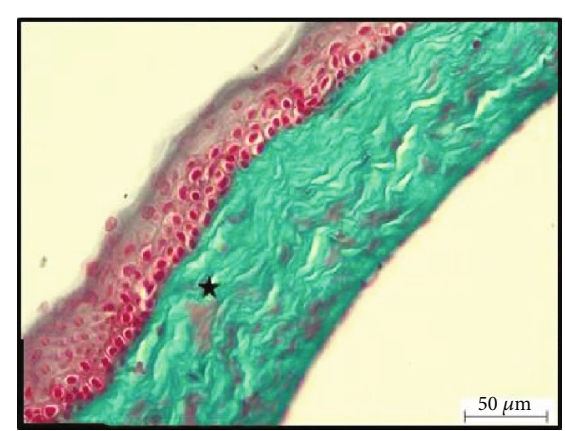

(a)

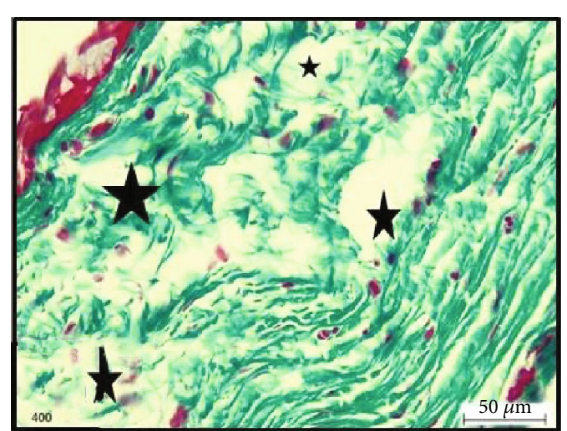

(b)

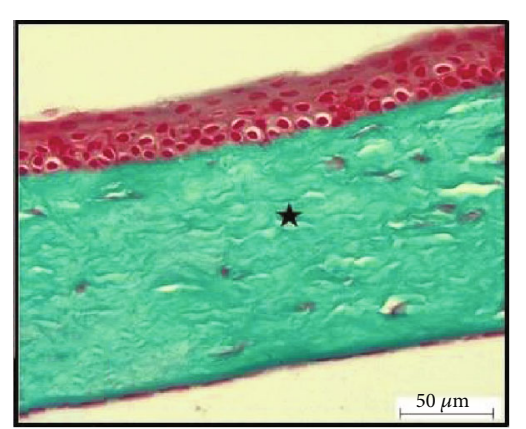

(c)

FIGURE 2: Photomicrographs of Masson trichrome sections in the rat cornea. (a) Group I (adult) section showing regularly arranged collagen fibers in the corneal stroma (asterisk) (Masson Trichrome $\times 400$ ). (b) Group IIa (aged) section showing disorganized widely separated collagen fibers in the corneal stroma (asterisk) (Masson Trichrome $\times 400$ ). (c) Group IIb (aged, PRP treated) section showing regularly arranged collagen fibers in the corneal stroma (asterisk) (Masson Trichrome $\times 400$ ).

Examination of HE stained sections of aged, PRP-treated group revealed an apparently normal corneal epithelium in comparison to the adult group with regular intact Bowman's membrane (Figure 1(g)). Cytoplasmic vacuolation was noticed in few epithelial cells. Corneal stroma had regularly arranged collagen fibers with few spaces and keratocytes in between. Endothelial cells and Descemet's membrane were apparently normal (Figures 1(h) and 1(i)).

3.2. Masson Trichrome Staining Results. Examination of Masson trichrome stained sections of the adult group revealed regularly arranged collagen bundles in the stroma (Figure 2(a)). However, Masson's trichrome stained cornea sections of the aged group showed disorganized, interrupted, and widely separated stromal collagen fibers (Figure 2(b)). On the other hand, Masson's trichrome stained cornea sections from aged, PRP-treated group showed regularly arranged collagen fibers (Figure 2(c)).

3.3. Immunohistochemical Results. Immunohistochemically, caspase-3 stained cornea sections from the adult group showed a weak immune reaction in the corneal epithelium nuclei (Figure 3(a)). In the aged group, immunohistochemically stained sections of caspase- 3 showed a strong immune reaction in nuclei of corneal epithelium and some keratocytes (Figure 3(b)), but in the aged, PRP-treated group, immunohistochemically stained sections showed a weak immune reaction in the corneal epithelium (Figure 3(c)).

3.4. Semithin Sections. Examination of the semithin sections of rat cornea from the adult group showed the three layers of corneal epithelium arranged as the inner basal columnar cells, intermediate multilayered polygonal cells, and the outer squamous layer. Bowman's membrane appeared as a thin regular membrane that basal cells resting on (Figure 4(a)). Avascular stroma appeared with regularly arranged collagen lamella with spindle-shaped keratocytes in between (Figures 4(a) and 4(b)). Descemet's membrane layer appeared thick and homogenous with an inner single layer of flat endothelial cells resting on (Figure 4(b)).

However, the examination of semithin cornea sections of the aged group revealed that disorganization and sloughing of cells in the surface epithelium. Basal and intermediate layers showed pale vacuolated cytoplasm with deeply stained nuclei that some of them were pyknotic and others were shrunken. Bowman's membrane appeared irregular, thin, and difficult to be detected (Figure 4(c)). Irregular collagen fibers observed in corneal stroma accompanied by wide spacing, nuclear changes in keratocytes, and neovascularization (Figure 4(d)).

In the aged, PRP-treated group, semithin cornea sections showed apparently normal cornea histology in comparison to the control group. The corneal epithelium layer basal cells, the intermediate layer, and the superficial squamous layer appeared like control (Figure 4(e)). Stromal collagen fibers showed regularly arranged with keratocytes in-between. Neovascularization and inflammatory infiltration cannot be detected (Figures 4(e) and 4(f)). Descemet's membrane appeared homogenous and regular with endothelial cells arranged regularly on it (Figure 4(f)).

3.5. Ultrathin Sections. Electron microscopic examination of the rat cornea from the adult group revealed histological structure. The corneal epithelium appeared to be formed of a basal layer of columnar cells with a euchromatic nucleus that was resting on the basement membrane (Bowman's membrane) (Figure 5(a)). The intermediate cell layer and the superficial layer appeared attached with numerous electron-dense desmosomes with narrow intercellular spaces. These cells showed few cytoplasmic organelles (Figure 5(b)). The corneal stroma was formed of regularly arranged collagen fibers with keratocytes in-between. The keratocytes were spindle-shaped cells with an oval nucleus, prominent nucleolus, and scanty cytoplasm (Figure 5(c)). Descemet's membrane appeared as an acellular thick homogenous electron-dense layer. A single layer of endothelial cells with flat electron-dense nuclei and scanty cytoplasm was resting on the Descemet's membrane (Figure 5(d)).

Ultrastructural examination of the aged group corneal sections revealed marked disfigurement of normal corneal ultrastructural. The corneal epithelium showed multiple cytoplasmic vacuolations, focal separation, and widening of the intercellular spaces with loss of desmosomal junctions. Corneal epithelial cells showed disfigurement in their shapes. 


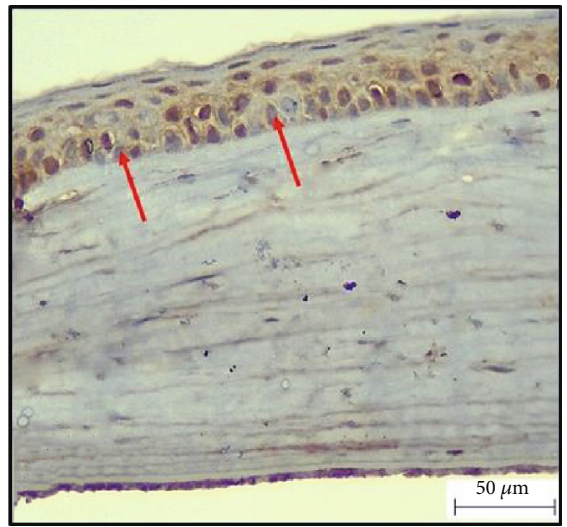

(a)

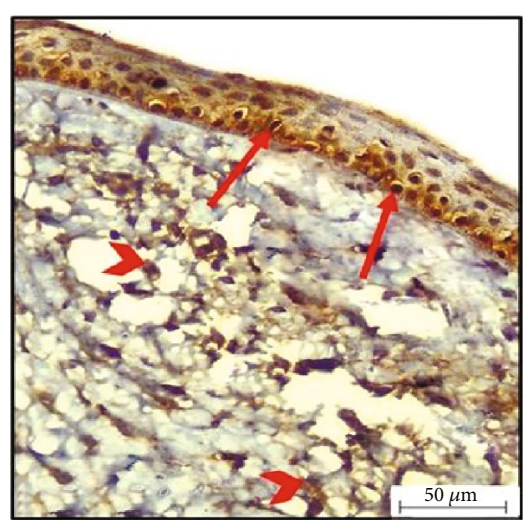

(b)

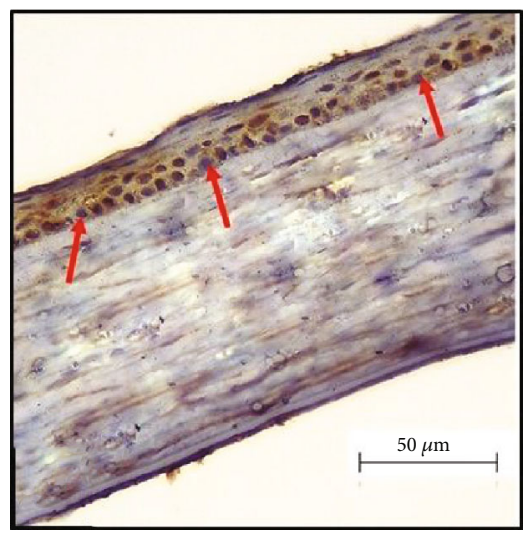

(c)

FIGURE 3: Photomicrographs of caspase-3 sections in the rat cornea. (a) Group I (adult) section showing weak immune reaction in the corneal epithelium nuclei (arrow) (caspase-3 $\times 400$ ). (b) Group IIa (aged) showing a strong immune reaction in nuclei of the corneal epithelium (arrow) and some keratocytes (arrowhead) (caspase-3 $\times 400$ ). (c) Group IIb (aged, PRP treated) section showing weak immune reaction in the corneal epithelium nuclei (arrow) (caspase- $3 \times 400)$.

They appeared with irregular nuclear membrane, shrunken hyperchromatic nucleus, and swollen mitochondria (Figure 6(a)). Corneal stroma showed irregularly arranged collagen fibers with wide spaces. Degenerated keratocytes appeared with shrunken nuclei, degenerated mitochondria, and cytoplasmic vacuolation (Figure 6(b)). Endothelial cells appeared with a degenerated electron-dense nucleus and shrunken mitochondria with cytoplasmic vacuolation (Figure 6(c)).

Moreover, the ultrastructure examination of the corneal section of the aged, PRP-treated group revealed apparently like the control group. However, the corneal epithelium showed cytoplasmic vacuolation with a slight widening of intercellular spaces (Figure 6(d)). The stroma appeared almost normal with narrow spaces, regularly arranged collagen fibers, and keratocytes with a euchromatic nucleus and scanty cytoplasm (Figure 6(e)). Descemet's membrane appeared thick homogenous with normal endothelial cells with an electron-dense elongated nucleus. Few small vacuoles were observed in a few endothelial cells (Figure 6(f)).

3.6. Morphometric and Biochemical Results. Morphometric and biochemical results from groups Ia, Ib, and Ic showed similar findings with no statistical differences.

3.6.1. The Thickness of the Corneal Epithelium. In group Ia (adult rats), the corneal epithelium thickness was $37.33 \pm$ $1.966 \mu \mathrm{m}$ (mean $\pm \mathrm{SD}$ ). In group IIa (aged rats), it was $19.083 \pm 2.835 \mu \mathrm{m}$. It showed a highly statistically significant decrease when compared to group Ia $(p<0.001)$. In group IIb (aged, PRP-treated rats), it was $35.667 \pm 3.326 \mu \mathrm{m}$ where it showed a highly statistically significant increase when compared to group IIa $(p<0.05)$ (Figure $7(a)$ ).

3.6.2. The Thickness of Descemet's Membrane. In group Ia (adult rats), the Descemet's membrane thickness was 7.378 $\pm 0.819 \mu \mathrm{m}$ (mean $\pm \mathrm{SD}$ ). In group IIa (aged rats), it was $9.978 \pm 0.335 \mu \mathrm{m}$. It showed a highly statistically significant increase when compared to group Ia $(p<0.001)$. In group
IIb (aged, PRP-treated rats), it was $6.708 \pm 0.548 \mu \mathrm{m}$ where it showed a highly statistically significant decrease when compared to group IIa $(p<0.05)$ (Figure $7(\mathrm{~b}))$.

3.6.3. Area of the Percentage of Caspase-3 Positive Cells. In group Ia (adult rats), the percentage of caspase- 3 positive cells was 8.166 \pm 1.169 (mean \pm SD). In group IIa (aged rats), it was $38.66 \pm 7.146$. It showed a highly statistically significant increase when compared to group Ia $(p<0.001)$. In group IIb (aged, PRP-treated rats), it was $10.33 \pm 1.639 \mu \mathrm{m}$ where it showed a highly statistically significant decrease when compared to group IIa $(p<0.05)$ (Figure $7(c))$.

3.6.4. Malondialdehyde (MDA) Level. In group Ia (adult rats), the MDA level was $0.4453 \pm 0.175 \mathrm{nmol} / \mathrm{mg}$ (mean $\pm \mathrm{SD})$. In group IIa (aged rats), it was $3.323 \pm 0.717 \mathrm{nmol} / \mathrm{mg}$. It showed a highly statistically significant increase when compared to group Ia $(p<0.001)$. In group IIb (aged, PRPtreated rats), it was $1.0015 \pm 0.553 \mathrm{nmol} / \mathrm{mg}$ where it showed a statistically significant decrease when compared to group IIa $(p<0.05)$ (Figure 8(a)).

3.6.5. Glutathione Peroxidase Activity (GPx). In group Ia (adult rats), GPx level was $40.795 \pm 2.06 \mathrm{U} / \mathrm{mg}$ (mean $\pm \mathrm{SD}$ ). In group IIa (aged rats), it was $35.403 \pm 2.097 \mathrm{U} / \mathrm{mg}$. It showed a statistically significant decrease when compared to group Ia $(p<0.05)$. In group IIb (aged, PRP-treated rats), it was $39.57 \pm 2.04 \mathrm{U} / \mathrm{mg}$ where it showed a statistically significant increase when compared to group IIa $(p<0.05)$ (Figure 8(b)).

3.6.6. Superoxide Dismutase (SOD). In group Ia (adult rats), the SOD level was $20.4017 \pm 1.8209 \mathrm{U} / \mathrm{mg}$ (mean $\pm \mathrm{SD})$. In group IIa (aged rats), it was $14.098 \pm 1.997 \mathrm{U} / \mathrm{mg}$. It showed a highly statistically significant decrease when compared to group Ia $(p<0.05)$. In group IIb (aged, PRP-treated rats), it was $18.273 \pm 1.566 \mathrm{U} / \mathrm{mg}$ where it showed a statistically significant increase when compared to group IIa $(p<0.05)$ (Figure 8(c)). 


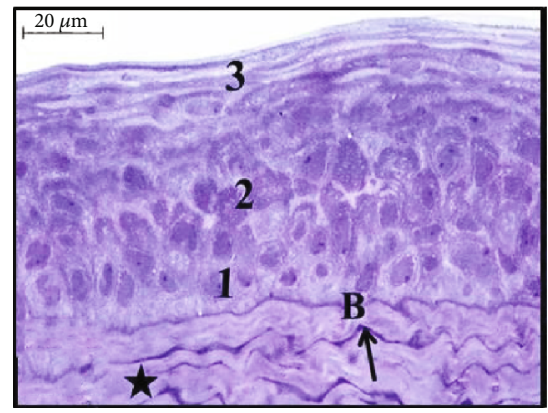

(a)

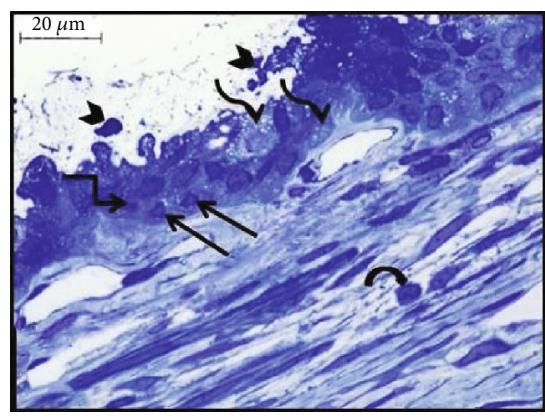

(c)

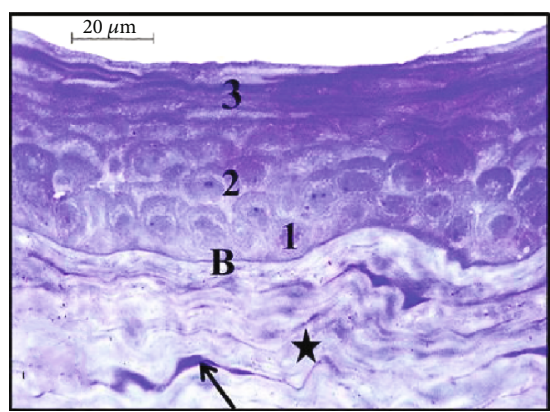

(e)

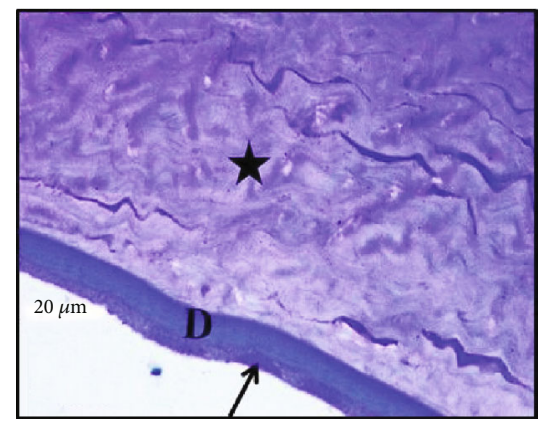

(b)

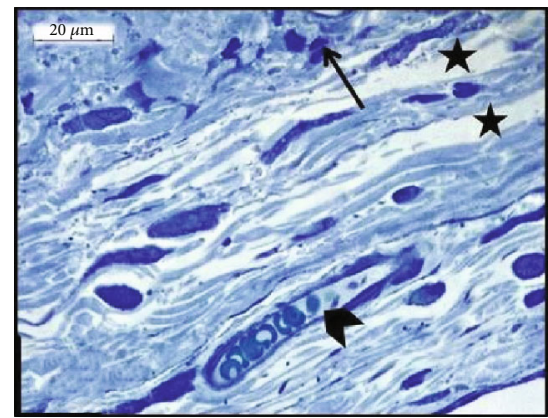

(d)

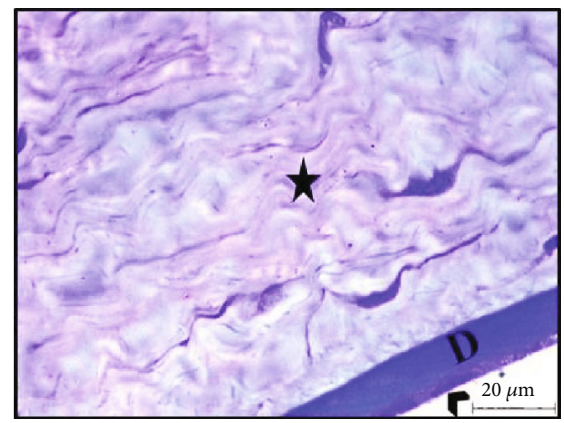

(f)

FIGURE 4: Photomicrographs of semithin sections in the rat cornea showing group I (adult): (a) layers of corneal epithelium: basal layer (1), the intermediate layer (2), and the superficial layer (3) that resting on regular Bowman's membrane (B). The collagen bundles of avascular stroma (asterisk) with keratocytes in between (arrow) (Toluidine blue $\times 1000)$; (b) thick homogenous Descemet's membrane layer (D) with a layer of flat endothelial cells resting on its inner surface (arrow) and stromal collagen fibers (asterisk) (Toluidine blue $\times 1000$ ). Group IIa (aged): (c) detached epithelial cells (arrowhead), pale vacuolated cytoplasm of basal, and intermediate cell layers (wavy arrow) with deeply stained nuclei that some of them are pyknotic (angular arrow) and others are shrunken (arrow). Notice the eosinophils (curved arrow) in the corneal stroma (Toluidine blue $\times 1000$ ); (d) irregular arranged widely spaced collagen bundles (asterisk), the neovascularization (arrowhead), and shrunken nuclei of keratocytes (arrow) (Toluidine blue $\times 1000$ ). Group IIb (aged, PRP treated): (e) layers of corneal epithelium arranged as an inner basal layer (1), the intermediate layer (2), and outer superficial layer (3). Notice the regular Bowman's membrane (B) and collagen bundles of avascular stroma (asterisk) with keratocytes (arrow) (Toluidine blue $\times 1000$ ); (f) corneal stroma collagen fibers (asterisk) and homogenous Descemet's membrane (D) with a single layer of flat endothelial cells (arrowhead) (Toluidine blue $\times 1000)$.

\section{Discussion}

The cornea is the primary refracting component of the visual system, and it is a part of the anterior segment of the eye that acts like a shield to keeps clear vision and protects against loss of fluids and pathogen invasion [22].

Aging is an intrinsic complex process. It is accompanied by a progressive loss of function and an increased mortality rate [23]. Aging is accompanied by the free radicals induced alterations of properties of the cell membrane including decreased fluidity, alteration in the electron transport chain complexes activities, and mitochondrial failure [24].

Oxidative stress plays an important role in the development and progression of the aging process which occurred due to an imbalance between free radicals and the antioxidants system $[23,24]$. In the eye, oxidative damage can result xin molecular changes that contribute to the development of age-related diseases such as cataracts and glaucoma [25].

Reactive oxygen species (ROS) may be a by-product of cellular aerobic metabolism or signaling molecules of stress 


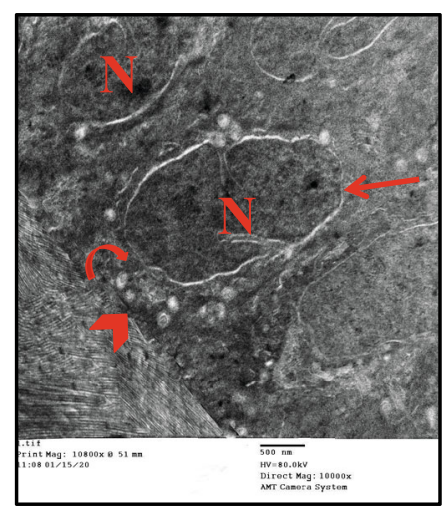

(a)

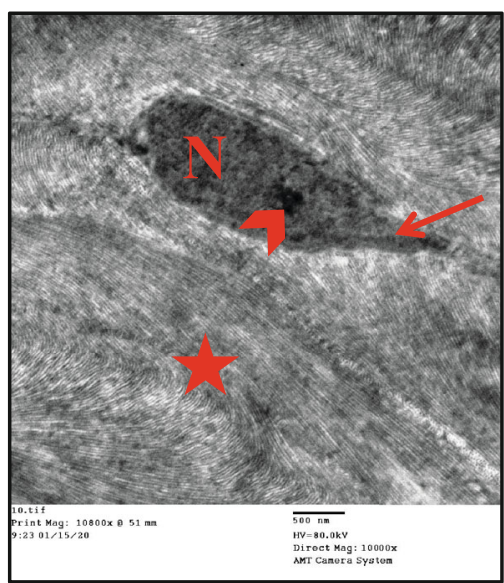

(c)

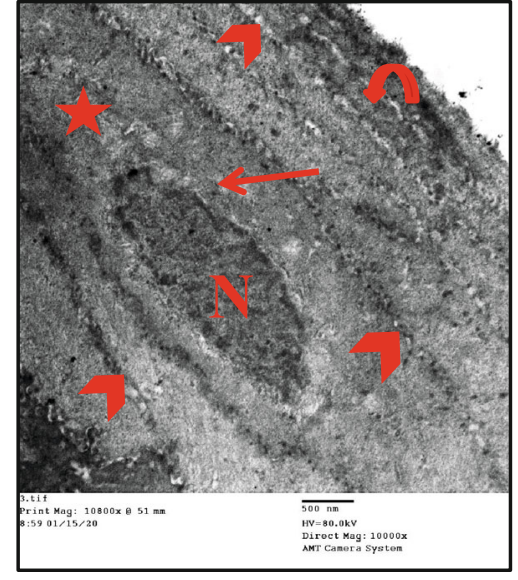

(b)

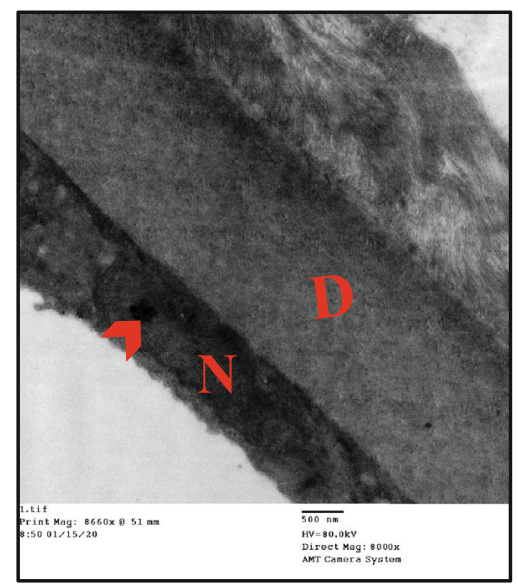

(d)

Figure 5: Photomicrographs of transmission electron micrograph sections in the rat cornea of group I (adult) showing (a) a single layer of basal columnar cells of the corneal epithelium with euchromatic nucleus $(\mathrm{N})$, regular nuclear membrane (arrow), and mitochondria (curved arrow) resting on Bowman's membrane (arrowhead) (Uranyl acetate and lead citrate $\times 10000$ ); (b) the polygonal cells of the intermediate layer of corneal epithelium with oval nuclei $(\mathrm{N})$, mitochondria (arrow), and the squamous cells of the superficial layer with a flat nucleus (curved arrow), numerous electron-dense desmosomes (arrowhead), and narrow intercellular spaces (asterisk) (Uranyl acetate and lead citrate $\times 10000$ ); (c) avascular corneal stroma with regularly arranged collagen fibers (asterisk) and spindle-shaped keratocytes with an oval nucleus $(\mathrm{N})$, prominent nucleus (arrowhead), and scanty cytoplasm (arrow) (Uranyl acetate and lead citrate $\times 10000)$; (d) Descemet's membrane as an acellular homogenous electron-dense layer (D) and a single layer of endothelial cells with a flat electron-dense nucleus $(\mathrm{N})$, with a prominent nucleolus (arrowhead) (Uranyl acetate and lead citrate $\times 8000$ ).

in response to cellular damage $[25,26]$. The production of ROS has to be balanced with reducing agents and antioxidant enzymes which protect the tissues against oxidative stress. However, when their production exceeds the antioxidant capacity, they induce damage to cell components such as DNA, proteins, and lipids [25].

The oxidative stress intensity may be estimated by the measurement of malondialdehyde (MDA) [26]. MDA is the main product of free radicals and an indicator of lipid peroxidation which is considered as a biomarker of aging [27, 28]. In the present study, there is a highly statistically significant increase in the MDA level in the aged rat group in comparison to the adult rat group.

On the contrary, the main reducing system in the eye is the glutathione system. Glutathione peroxidase (GPx) is a family of enzymes that plays an important role in the protection from oxidative damage as it leads to the oxidation of glutathione (GSH) to oxidized glutathione (GSSG) [25, 29]. A statistically significant decrease in GPx level was detected in the aged rat group in comparison to the adult rat group.

Additionally, superoxide dismutase (SOD) is an antioxidant enzyme that plays an important protective role against ROS and is considered as the first line of antioxidant defense $[25,26]$. Free radicals are converted by SOD into hydrogen peroxide which can form a highly reactive hydroxyl ion that reacts with phospholipids in cell membranes and proteins [30]. A highly statistically significant decrease in the SOD level was detected in the aged rat group in comparison to the adult rat group.

In the current study, the histological and ultrastructural examination of the corneal sections of the aged rat's group revealed marked structural changes that affected all the corneal layers. 


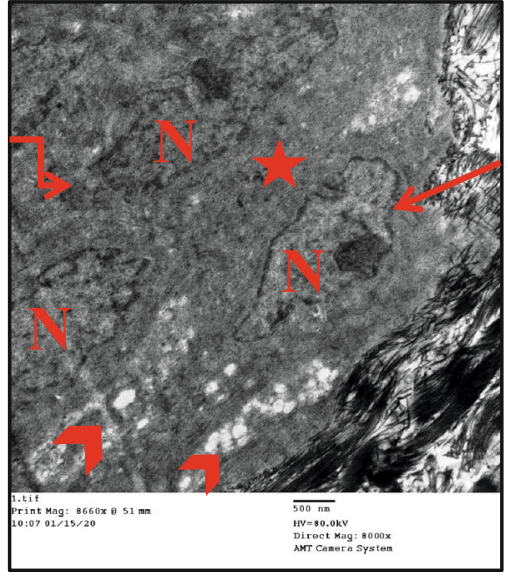

(a)

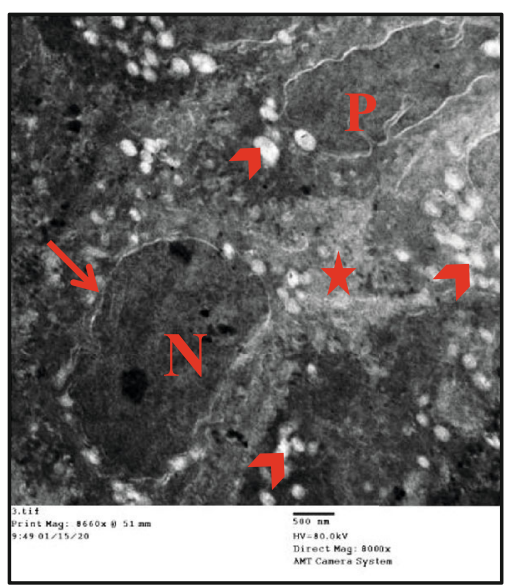

(d)

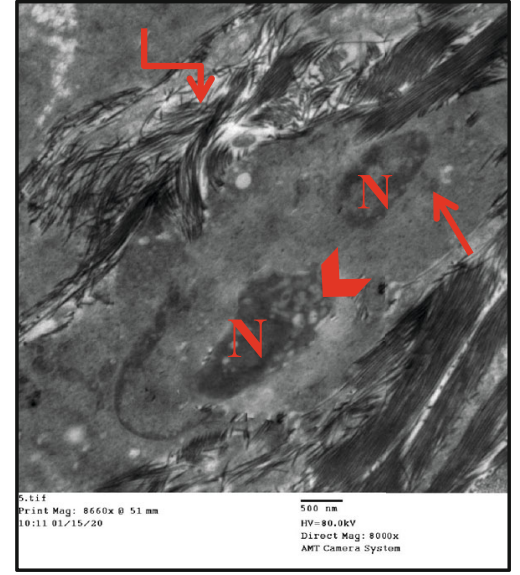

(b)

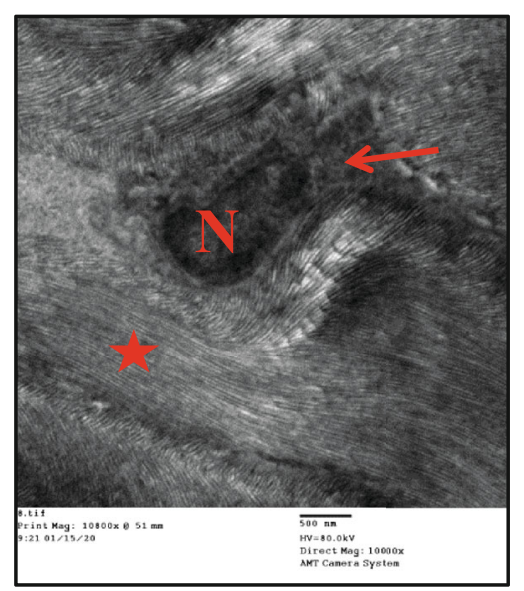

(e)

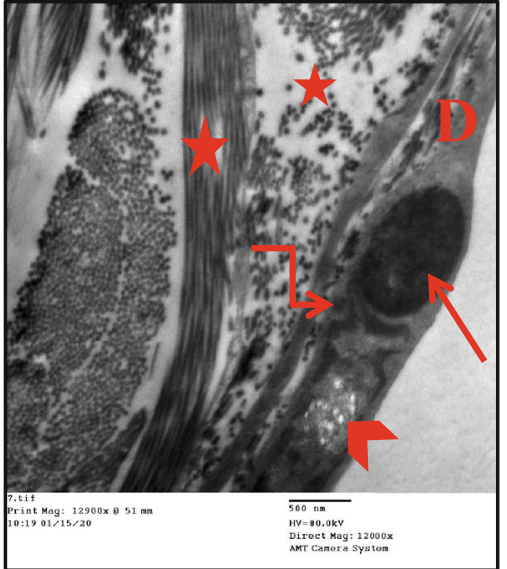

(c)

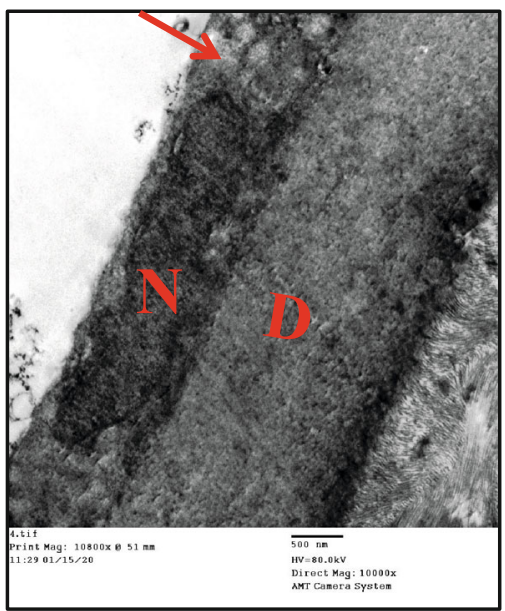

(f)

FIGURE 6: Photomicrographs of transmission electron micrograph sections in the rat cornea showing group IIa (aged): (a) corneal epithelial cells (basal and intermediate cells) with irregular nuclear membrane (arrow), shrunken hyperchromatic nucleus (N), and swollen mitochondria (angular arrow). Notice the multiple cytoplasmic vacuolations (arrowhead) and widening of the intercellular spaces with loss of desmosomal junctions (asterisk) (Uranyl acetate and lead citrate $\times 8000$ ); (b) corneal stroma with irregularly arranged collagen bundles (angular arrow) and degenerated keratocytes with shrunken nuclei (N), shrunken mitochondria (arrow), and cytoplasmic vacuolation (arrowhead) (Uranyl acetate and lead citrate $\times 8000$ ); (c) collagen fibers with different directions (asterisk), Descemet's membrane (D), and endothelial cell with degenerated electron-dense nucleus (arrow), shrunken mitochondria (angular arrow), and cytoplasmic vacuolation (arrowhead) (Uranyl acetate and lead citrate $\times 12000$ ). Group IIb (aged, PRP treated): (d) euchromatic nucleus of the basal columnar cell $(\mathrm{N})$ with regular nuclear membrane (arrow) and euchromatic nucleus of the polygonal of the intermediate layer (P). Notice the cytoplasmic vacuolation (arrowhead) with a slight widening of intercellular spaces (asterisk) (Uranyl acetate and lead citrate $\times 8000$ ); (e) regularly arranged collagen fibers of the corneal stroma (asterisk) and spindle-shaped keratocytes with an oval nucleus $(\mathrm{N})$ and scanty cytoplasm (arrow) (Uranyl acetate and lead citrate $\times 10000$ ); (f) regular homogenous Descemet's membrane (D) and electron-dense elongated nucleus of endothelial cells $(\mathrm{N})$ with small vacuoles (arrow) (Uranyl acetate and lead citrate $\times 10000$ ).

Focal discontinuity with sloughing of the surface epithelium was detected accompanied by a highly statistically significant decrease in the corneal epithelium of the aged group in comparison to the adult group. Thinning of the corneal epithelium might be due to a decrease in the capacity of corneal epithelial cells to proliferate [15]. Aseta et al. [31] reported that the epithelial thinning and cell loss detected in case of eye closure may be secondary to hypoxia.

Elwan and Kassab [18] demonstrate corneal epithelial thinning in the case of capecitabine-induced corneal toxicity that might be due to cellular degeneration and slow rate of cellular regeneration and proliferation.
Faragher et al. [8] mentioned that aging is associated with a reduction in the epithelial barrier function and increased epithelial permeability with changes in the distribution of integrin subunits.

Meanwhile, the epithelial cells of the aged cornea had shrunken hyperchromatic nuclei, irregular nuclear membrane, and swollen mitochondria with pale vacuolated cytoplasm. Elwan and Kassab [18] demonstrated pyknotic nuclei in the corneal epithelial cells in the case of corneal toxicity. These findings were interpreted as apoptosis by the authors.

Aseta et al. [31] explained the cytoplasmic vacuolation observed in case of eye closure as a reflection for a form of 


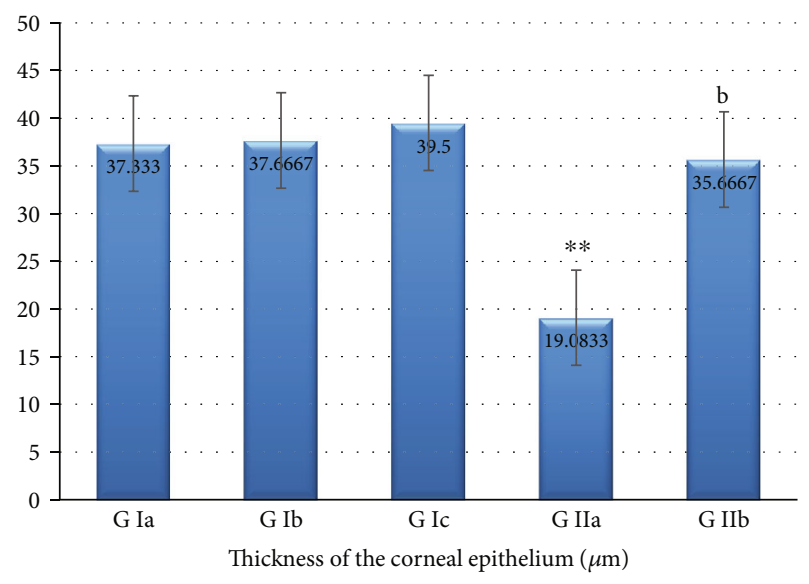

(a)

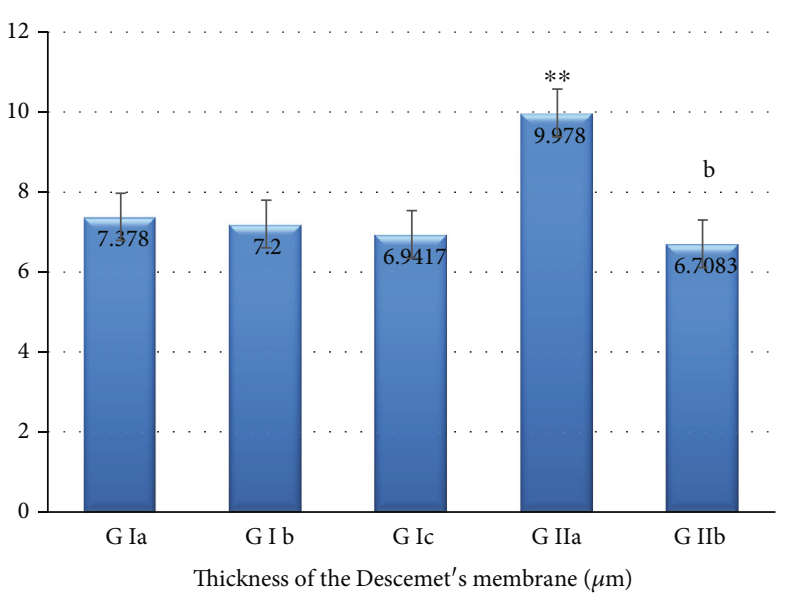

(b)

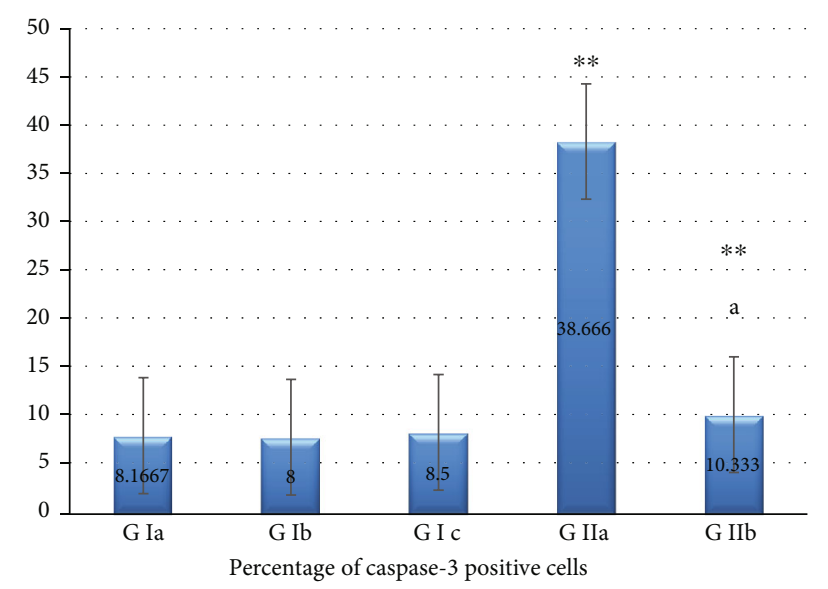

(c)

FIGURE 7: Effect of PRP treatment on the structure of the cornea in the studied age groups of animals. All data represented as mean \pm SEM. Data were analyzed by one-way ANOVA using Tukey's post hoc test. (a) Histogram showing the thickness of the corneal epithelium ( ${ }^{* *}$, highly statistically significant versus adult group) (b, statistically $(p<0.05)$ versus aged group $(p<0.001)$ ). (b) Histogram showing the thickness of the Descemet's membrane $(* *$, highly statistically significant versus adult group) (b, statistically $(p<0.05)$ versus aged group $(p<0.001))$. (c) Histogram showing percentage of caspase-3 positive cells $\left({ }^{* *}\right.$, highly statistically significant versus adult group) (a, statistically $(p<0.05)$ versus aged group $(p<0.001))$.

programmed cell death (type III cytoplasmic cell death) that is triggered by insulin-like growth factor or tumor necrosis factor.

Moreover, Bowman's membrane in the aged cornea was very thin and irregular that associated with widening in intercellular spaces and loss of desmosomal junctions. Ebrahim et al. [17] mentioned similar findings in the case of corneal alkali burns in rats due to corneal epithelial cells desquamation and necrosis. Also, Aseta et al. [31] detected these changes in case of eye closure and clarified that any changes occurred in the barrier function of the corneal epithelium will affect the integrity of the cornea that leads to an increased risk of infections, corneal diseases, and corneal damage.

In the current study, corneal stroma in the HE sections of the aged cornea showed various structural changes detected in the form of disarrangement and spacing in the collagen bundles with the appearance of neovascularization and inflammatory cellular infiltration. Halawa [15] observed in the stroma, wide separation of the corneal lamellae with invasion by blood vessels. She suggested that this will result in cornea edema and affect corneal transparency. Daxer et al. [32] reported that the spacing resulted from glycationinduced cross-linking and reduction of the molecular tilting angle within collagen fibrils.

The examination of the Masson trichrome stained section confirmed the stromal finding of HE sections. Corneal opacity and dry eye are a common complaint in old age caused by associated corneal edema due to widely spaced collagen lamella and reduction of sodium and potassiumdependent ATPase enzyme levels [2].

Elwan and Kassab [18] reported that stromal inflammatory cellular infiltration may be due to the release of interleukin $1 \beta$ (IL1 $\beta)$ from the injured corneal epithelial cells in the rat model of capecitabine-induced injury.

A healthy functioning cornea is avascular. The circulating vascular endothelial growth factor (VEGF) receptors play an important role in corneal avascularity through binding 


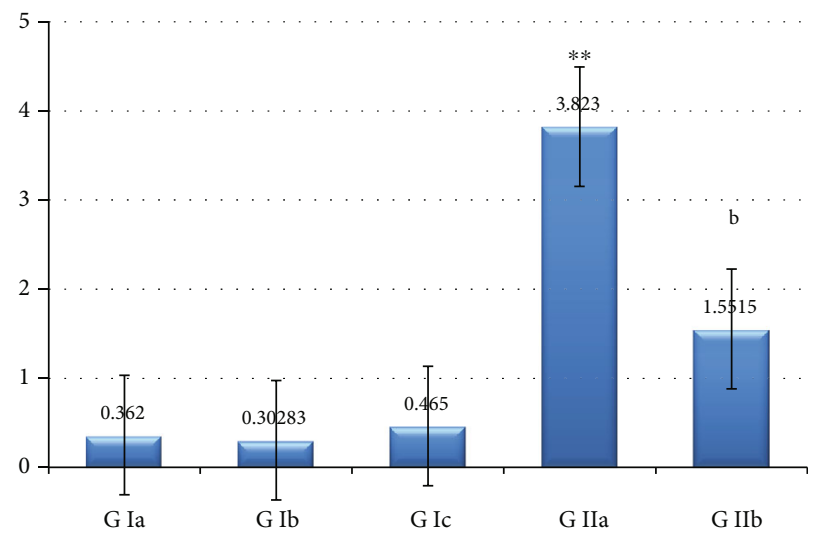

(a)

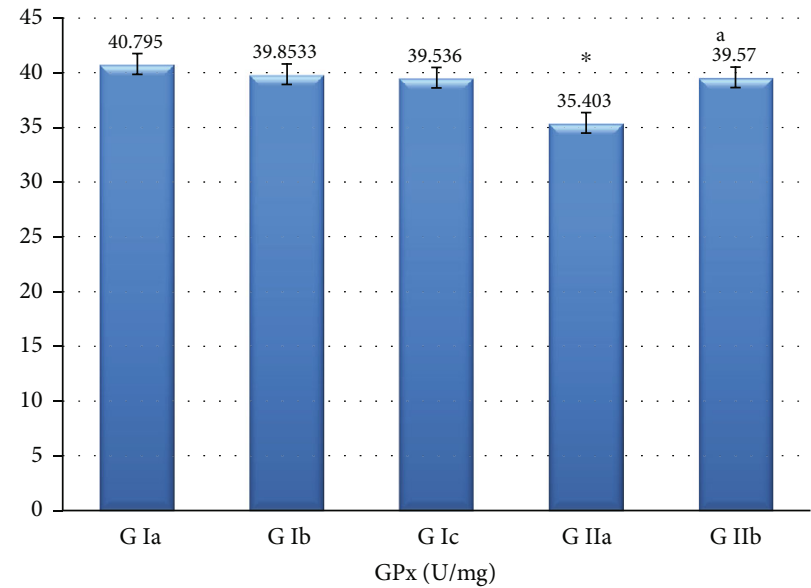

(b)

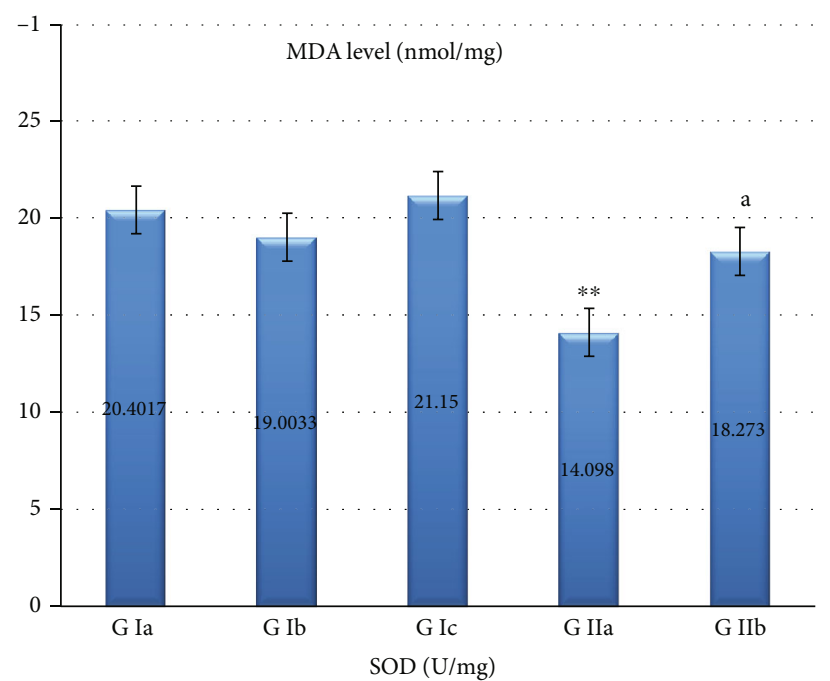

(c)

FIgURE 8: Effect of PRP treatment on the level of oxidative stress in the corneal tissue of the studied groups of animals. All data represented as mean \pm SEM. Data were analyzed by one-way ANOVA using Tukey's post hoc test. (a) Histogram showing the MDA level (**, highly statistically significant versus adult group) (b, statistically $(p<0.05)$ versus aged group $(p<0.001))$. (b) Histogram showing the GPx level $(*$, statistically $(p<0.05)$ versus adult group) (a, statistically $(p<0.05)$ versus aged group). (c) Histogram showing SOD level $(* *$, highly statistically significant versus adult group) (a, statistically $(p<0.05)$ versus aged group $(p<0.001)$ ).

with the free VEGF thereby making it unavailable for binding to receptors on cell membranes [33].

Faruk et al. [34] detected the neovascularization in histological and ultrastructural sections of a rat model of induced corneal alkali burn. Berthaut et al. [33] mentioned that the expression of VEGF receptors decreased with age in humans. On the other hand, Tendler et al. [35] revealed that neovascularization could be secondary to associated corneal inflammation and edema.

Corneal neovascularization is a major, sight-threatening complication of some ocular disorders. It results from an imbalance between angiogenic and antiangiogenic factors and is characterized by overproliferation, migration, and capillary tube formation by endothelial cells [36].

In the current study, keratocytes in aged cornea sections appeared with shrunken nuclei, shrunken mitochondria, and vacuolated cytoplasm. Keratocytes are considered as cor- neal fibroblasts that are involved in the process of inflammation [37]. Wang et al. [38] revealed the disarrangement of the collagen fibers due to keratocyte apoptosis.

This theory was supported by the results of caspase-3 immune-histochemical stained section examination which revealed a highly statistically significant increase in caspase3 positive cells in corneal epithelium and keratocytes.

Rhim et al. [39] mentioned a higher level of caveolin-1 was detected in the corneal epithelium of elderly patients than the young one that indicated an increased average of cell damage. Also, the increase of TGF-b in aged cornea reflecting an increase of apoptotic death [7].

On the other hand, a highly statistically significant increase in Descemet's membrane thickness was detected in the aged group in comparison to the adult group with areas of separation between the membrane and the corneal stroma. Swollen endothelial cells with a degenerated electron-dense 
nucleus, shrunken mitochondria, and cytoplasmic vacuolation were observed.

Descemet's membrane (DM) is the basement membrane of the corneal endothelium composed predominately of type VIII collagen. It plays a crucial role in endothelial cell differentiation and proliferation and structural integrity [40]. The corneal endothelial layer has an important function to maintain corneal transparency. Loss of endothelial cells leads to irreversible corneal edema [41].

Jun et al. [42] detected an age-dependent focal thickening in Descemet's membrane in mice. Roh et al. [43] demonstrated the thickening of Descemet's membrane with an electron microscopy examination. Mousa et al. [2] and Farid et al. [44] explained the changes in these layers resulted from the failure of the cross-linking of collagen with the degeneration of endothelial cells. Jun et al. [42] revealed that there is an aging-associated accumulation of the posterior, nonbanded portion to this layer.

PRP is an autologous serum that is known to be safe, effective, and powerful to repair tissue damage depending on its high concentration of platelets that contain a wide variety of growth factors $[45,11]$. PRP application has promising results in acute as well as chronic injuries [46]. Recently, PRP is used in many medical fields such as regenerative medicine, dental implants and prostheses, maxillofacial surgery, plastic, orthopedics, treatment diabetic ulcer, and dry eye [13, 47].

To the best of our knowledge, it has not been reported for the usage of PRP on spontaneous age-associated corneal changes.

In the current work, the examination of histological and ultrastructural corneal sections of the aged, PRP-treated group revealed a normal histological structure comparable to the adult group. It showed intact Bowman's membrane and arranged corneal epithelium in its three layers. Moreover, well-organized collagen lamella in the stroma with keratocytes situated in-between and continuous regular Descemet's membrane with regularly situated endothelial cells. However, ultrastructural examination revealed vacuolated cytoplasm of epithelial cells with wide intercellular spaces. A highly statistically significant increase and decrease in corneal epithelium thickness and Descemet's membrane thickness were detected, respectively.

Sharaf Eldin et al. [14] and Charalambidou et al. [48] detected similar findings after using PRP in a rat model of corneal alkali burn, and Tanidir et al. [49] reported the same in a rabbit model of corneal epithelium wound healing.

They explained that due to a high concentration of platelets that contained growth factors attached to the corneal surface and accelerate tissue repair mechanism. Zheng et al. [16] and Mansy et al. [50] mentioned that PRP played a dominant role in cell migration and proliferation.

Moreover, the examination of sections revealed a reduction in inflammatory cellular infiltration, vacuolation, and neovascularization. Çirci et al. [51] and Martini et al. [52] explained that PRP has a strong anti-inflammatory and antibacterial effect that reduce cellular inflammation markers. Lee et al. [45] reported that using PRP in treatment reduces inflammation and pain and enhances the healing process.
In the current study, the examination of caspase- 3 immunohistochemical stained sections shows a weak immune reaction and a highly statistically significant decrease in the aged, PRP-treated group in comparison to the aged group. Rah et al. [53] stated that PRP acts as an apoptosis regulatory messenger as it reduces the expression of ASK-1, a typical member of the mitogen-activated protein kinase kinase kinase (MAPKKK) family and the critical component in ROS-induced apoptosis.

Additionally, a statistically significant decrease in the MDA level was accompanied by a statistically significant increase in GPx and SOD levels. Rah et al. [53] and Chen et al. [54] mentioned that PRP had an antioxidant effect due to its content of numerous growth factors. These growth factors resulted in a decrease in oxidative stress and a reduction in reactive oxygen species. Also, these growth factors enhance the healing processes such as cell proliferation, differentiation, and regeneration of damaged tissues through increase nutrient influx and blood supply $[55,56]$.

\section{Conclusion}

The findings of the current study can highlight evidence about the efficient regenerative role of single subconjunctival injection of autologous PRP in ameliorating the age-related changes in rat's cornea. That can be concluded through amelioration of the redox state and restoration of the structure of a normal healthy cornea.

\section{Data Availability}

The data used to support the findings of this study are available upon request.

\section{Conflicts of Interest}

There is no conflict of interest.

\section{Acknowledgments}

The author would like to express my sincere appreciation to Professor Soheir Ibrahim Saleh, Professor of Anatomy and Embryology, Faculty of Medicine, Ain Shams University, for her guidance, support, and a great help in reviewing the study. Also, profound gratefulness for Ass. Professor Basma Hamdy Amin, Assistant Professor of Microbiology at the Regional Center for Mycology and Biotechnology, Al-Azhar University, for her professional preparation of electron microscopic samples.

\section{References}

[1] I. K. Gipson, "Age-related changes and diseases of the ocular surface and cornea," Investigative Opthalmology \& Visual Science, vol. 54, no. 14, pp. ORSF48-ORSF53, 2013.

[2] A. M. Mousa, A. el-Mahalaway, and R. el-Desoky, "The protective effects of sodium hyaluronate on corneal injuries induced by chlorinated water in adult male rabbits: A histological and immunohistochemical study," Egyptian Journal of Histology, vol. 41, no. 2, pp. 140-149, 2018. 
[3] L. Dvorscak and C. F. Marfurt, "Age-related changes in rat corneal epithelial nerve density," Investigative Ophthalmology and Visual Science, vol. 49, no. 3, pp. 910-916, 2008.

[4] P. N. Lewis, T. L. White, R. D. Young, J. S. Bell, C. P. Winlove, and K. M. Meek, "Three-dimensional arrangement of elastic fibers in the human corneal stroma," Experimental Eye Research, vol. 146, pp. 43-53, 2016.

[5] T. L. White, P. N. Lewis, R. D. Young et al., "Elastic microfibril distribution in the cornea: Differences between normal and keratoconic stroma," Experimental Eye Research, vol. 159, pp. 40-48, 2017.

[6] A. Meeney and H. S. Mudhar, "Histopathological reporting of corneal pathology by a biomedical scientist: the Sheffield Experience," Eye, vol. 27, no. 2, pp. 272-276, 2013.

[7] H. I. H. El-Sayyad, A. A. El-Mansi, M. S. Guida, and E. A. Mohammed, "Markers characterizing corneal damage during aging of rat," Journal of Advances in Chemistry, vol. 11, no. 5, pp. 3532-3539, 2015.

[8] R. G. A. Faragher, B. Mulholland, S. J. Tuft, S. Sandeman, and P. T. Khaw, "Aging and the cornea," The British Journal of Ophthalmology, vol. 81, no. 10, pp. 814-817, 1997.

[9] G. Vitályos, B. L. Kolozsvári, G. Németh et al., "Effects of aging on corneal parameters measured with Pentacam in healthy subjects," Scientific Reports, vol. 9, no. 1, pp. 3419-3419, 2019.

[10] M. Girgin, K. Binnetoglu, K. Duman et al., "Effects of platelet rich plasma on fascial healing in rats with fecal peritonitis," Acta Cirúrgica Brasileira, vol. 31, no. 5, pp. 314-319, 2016.

[11] H. A. Farghali, N. A. AbdElKader, H. O. AbuBakr et al., "Antimicrobial action of autologous platelet-rich plasma on MRSAinfected skin wounds in dogs," Scientific Reports, vol. 9, no. 12722, pp. 1-15, 2019.

[12] D. Chicharro-Alcántara, M. Rubio-Zaragoza, E. Damiá-Giménez et al., "Platelet rich plasma: New insights for cutaneous wound healing management," Journal of Functional Biomaterials, vol. 9, no. 1, p. 10, 2018.

[13] N. Salem, N. Helmi, and N. Assaf, "Renoprotective Effect of Platelet-Rich Plasma on Cisplatin-Induced Nephrotoxicity in Rats," Oxidative Medicine and Cellular Longevity, vol. 2018, Article ID 9658230, 10 pages, 2018.

[14] H. Sharaf eldin, M. Ibrahim, and N. Elswaidy, "A histological and immunohistochemical study of the effect of platelet- rich plasma on a corneal alkali burn in adult male albino rat," Egyptian Journal of Histology, vol. 42, no. 2, pp. 482-495, 2019.

[15] A. M. Halawa, "Age-Associated Changes in the Cornea, Lens and Retina of the Albino Rat Eye: A Histological and Immuno-Histochemical Study," The Egyptian Journal of Anatomy, vol. 34, no. 1, pp. 1-13, 2011.

[16] X. Zheng, H. Li, L. Du, Q. Gu, and H. Wang, "A rat model of proliferative vitreoretinopathy induced by RPE-J cells and platelet-rich plasma," Asian Biomed, vol. 3, no. 5, pp. 507515, 2009.

[17] N. Ebrahim, O. M. Mohammed, A. A. Dessouky, and D. S. A. Fatah, "The potential therapeutic effect of stem cells loaded on two different vehicles (amniotic membrane and platelet rich plasma gel) in experimentally induced corneal alkali burns in rats," Egyptian Journal of Histology, vol. 40, no. 4, pp. 405426, 2017.

[18] W. M. Elwan and A. A. Kassab, "The potential protective role of hesperidin against capecitabine-induced corneal toxicity in adult male albino Rat. Light and electron microscopic study," Egyptian Journal of Histology, vol. 40, no. 2, pp. 201-215, 2017.
[19] S. K. Suvarna, C. Layton, and J. D. Bancroft, Bancroft's Theory and Practice of Histological Techniques, Elsevier Health sciences, Churchill Livingstone, 7th edition, 2013.

[20] S. Kara, B. Gencer, T. Karaca et al., "Protective effect of hesperetin and naringenin against apoptosis in ischemia/reperfusioninduced retinal injury in rats," The Scientific World Journal, vol. 2014, Article ID 797824, 8 pages, 2014.

[21] J. J. Bozzala and D. Russell Lonnie, Electron Microscopy: Principles and Techniques for Biologists, Jones and Bartlett Publishers, Sudbury, massachusetts, 2nd edition, 2002.

[22] B. J. Blackburn, M. W. Jenkins, A. M. Rollins, and W. J. Dupps, "A review of structural and biomechanical changes in the cornea in aging, disease, and photochemical crosslinking," Frontiers in Bioengineering and Biotechnology, vol. 7, no. 6, pp. 116, 2019.

[23] H. Cui, Y. Kong, and H. Zhang, "Oxidative stress, mitochondrial dysfunction, and aging," Journal of Signal Transduction, vol. 2012, 13 pages, 2012.

[24] B. L. Tan, M. E. Norhaizan, W. P. P. Liew, and H. Sulaiman Rahman, "Antioxidant and oxidative stress: a mutual interplay in age-related diseases," Frontiers in Pharmacology, vol. 9, pp. 1-28, 2018.

[25] L. F. Hernandez-Zimbron, R. Gulias-Cañizo, M. F. Golzarri, B. E. Martínez-Baéz, H. Quiroz-Mercado, and R. GonzalezSalinas, "Molecular age-related changes in the anterior segment of the eye," Journal of Ophthalmology, vol. 2017, 8 pages, 2017.

[26] M. Kozakiewicz, M. Kornatowski, O. Krzywińska, and K. Kędziora-Kornatowska, "Changes in the blood antioxidant defense of advanced age people," Clinical Interventions in Aging, vol. Volume 14, pp. 763-771, 2019.

[27] N. Muralidharan, T. Bhat, and S. Kumari, "Section: Medicine A study on effect of ageing on the levels of total antioxidant and lipid peroxidation Section," Medicine, vol. 4, no. 12, pp. 8-10, 2017.

[28] M. Balci, E. Devrim, and I. Durak, "Effects of mobile phones on oxidant/antioxidant balance in cornea and lens of rats," Current Eye Research, vol. 32, no. 1, pp. 21-25, 2009.

[29] E. Mohamed and M. Ali, "Autologus serum and sodium hyaluronate role in alkali corneal burn healing," Journal of The Arab Society for Medical Research, vol. 13, pp. 39-44, 2018.

[30] I. Liguori, G. Russo, F. Curcio et al., "Oxidative stress, aging, and diseases," Clinical Interventions in Aging, vol. Volume 13, pp. 757-772, 2018.

[31] F. B. Aseta, P. M. Mwachaka, P. O. Odula, and A. K. Malek, "Histomorphological changes in the cornea of the rat following monocular eyelid closure," Anatomy, vol. 10, no. 2, pp. 87-93, 2016.

[32] A. Daxer, K. Misof, B. Grabner, A. Ettl, and P. Fratzl, "Collagen fibrils in the human corneal stroma: structure and aging," Investigative Ophthalmology \& Visual Science, vol. 39, no. 3, pp. 644-648, 1998.

[33] A. Berthaut, P. Mirshahi, N. Benabbou et al., "Vascular endothelial growth factor receptor-1 (VEGFR-1) expression in human corneal fibroblast decreased with age," Molecular Vision, vol. 15, pp. 1997-2007, 2009.

[34] E. M. Faruk, A. El Mansy, A. M. Al Shazly, and N. M. Taha, "Light and electron microscopic study of the antiinflammatory role of mesenchymal stem cell therapy in restoring corneal alkali injury in adult albino rats," Journal of Stem Cell Biology and Transplantation, vol. 1, no. 1, pp. 1-11, 2018. 
[35] Y. Tendler, R. Pokroy, A. Panshin, and G. Weisinger, "P53 protein subcellular localization and apoptosis in rodent corneal epithelium cell culture following ultraviolet irradiation," International Journal of Molecular Medicine, vol. 31, no. 3, pp. 540-546, 2013.

[36] L. Yang, Y. Wang, Q. Zhou et al., "Inhibitory effects of polysaccharide extract from Spirulina platensis on corneal neovascularization," Molecular Vision, vol. 15, pp. 1951-1961, 2009.

[37] J. Ma, D. Zhou, M. Fan et al., "Keratocytes create stromal spaces to promote corneal neovascularization via MMP13 expression," Investigative Ophthalmology \& Visual Science, vol. 55, no. 10, pp. 6691-6703, 2014.

[38] M. Wang, M. Li, P. Yan, Q. Luo, Y. Zhang, and Z. Du, “The Effect of High Intensity Focused Ultrasound Keratoplasty on Rabbit Anterior Segment," Journal of Ophthalmology, vol. 2017, Article ID 6067890, 10 pages, 2017.

[39] J. H. Rhim, J. H. Kim, E.-J. Yeo, J. C. Kim, and S. C. Park, "Caveolin-1 as a novel indicator of wound-healing capacity in aged human corneal epithelium," Molecular Medicine, vol. 16, no. 11-12, pp. 527-534, 2010.

[40] T. B. McKay, S. Priyadarsini, and D. Karamichos, "Mechanisms of collagen crosslinking in diabetes and keratoconus," Cells, vol. 8, no. 10, p. 1239, 2019.

[41] L. Harkema, S. A. Youssef, and A. de Bruin, "Pathology of mouse models of accelerated aging," Veterinary Pathology, vol. 53, no. 2, pp. 366-389, 2016.

[42] A. S. Jun, S. Chakravarti, H. F. Edelhauser, and M. Kimos, "Aging changes of mouse corneal endothelium and Descemet's membrane," Experimental Eye Research, vol. 83, no. 4, pp. 890-896, 2006.

[43] D. S. Roh, Y. Du, M. L. Gabriele, A. R. Robinson, L. J. Niedernhofer, and J. L. Funderburgh, "Age-related dystrophic changes in corneal endothelium from DNA repairdeficient mice," Aging Cell, vol. 12, no. 6, pp. 1122-1131, 2013.

[44] E.-K. W. F. MA, F. K. Mansour, and A. S. Zolfakar, "Experimentally induced diabetic keratopathy in albino rats and the possible protective role of ginger," Journal of American Science, vol. 9, no. 12, pp. 206-220, 2013.

[45] J. H. Lee, M. J. Kim, S. W. Ha, and H. K. Kim, “Autologous platelet-rich plasma eye drops in the treatment of recurrent corneal erosions," Korean Journal of Ophthalmology, vol. 30, no. 2, pp. 101-107, 2016.

[46] P. Amable, R. B. Carias, M. V. Teixeira et al., "Platelet-rich plasma preparation for regenerative medicine: optimization and quantification of cytokines and growth factors," Stem Cell Research \& Therapy, vol. 4, no. 3, p. 67, 2013.

[47] B. J. Abdullah, N. Atasoy, and A. K. Omer, "Evaluate the effects of platelet rich plasma (PRP) and zinc oxide ointment on skin wound healing," Annals of Medicine and Surgery, vol. 37, pp. 30-37, 2019.

[48] G. Charalambidou, E. Kofidou, N. Papaioannou, V. Karampatakis, and A. Komnenou, "The effect of intrastromal PRP and oral doxycycline in corneal wound healing after alkali burn," Journal of the Hellenic Veterinary Medical Society, vol. 69, no. 4, pp. 1213-1225, 2019.

[49] S. T. Tanidir, N. Yuksel, O. Altintas, D. K. Yildiz, E. Sener, and Y. Caglar, "The effect of subconjunctival platelet-rich plasma on corneal epithelial wound healing," Cornea, vol. 29, no. 6, pp. 664-669, 2010.

[50] A. E. Mansy, N. E.-E. El-Azab, and A. M. El-Mahalaway, "Histological and immunohistochemical study of the potential healing effects of glucosamine and platelets rich plasma on experimentally induced oral mucositis in adult male rats," Journal of Histology and Histopathology, vol. 4, no. 1, p. 12, 2017.

[51] E. Çirci, Y. E. Akman, E. Sükür, E. R. Bozkurt, T. Tüzüner, and Y. Öztürkmen, “The Impact of platelet-rich plasma (PRP) injection timing on the healing of Achilles tendon injuries in rats," Acta Orthopaedica et Traumatologica Turcica, vol. 50, no. 3, pp. 366-372, 2015.

[52] L. I. Martini, A. G. Via, C. Fossati, F. Randelli, P. Randelli, and D. Cucchi, "Single platelet-rich plasma injection for early stage of Osteoarthritis of the knee," Joints, vol. 5, no. 1, pp. 002-006, 2017.

[53] D. K. Rah, H. J. Min, Y. W. Kim, and Y. W. Cheon, "Effect of platelet-rich plasma on ischemia-reperfusion injury in a skin flap mouse model," International Journal of Medical Sciences, vol. 14, no. 9, pp. 829-839, 2017.

[54] N.-F. Chen, C.-S. Sung, Z.-H. Wen et al., "Therapeutic effect of platelet-rich plasma in rat spinal cord injuries," Frontiers in Neuroscience, vol. 12, pp. 1-12, 2018.

[55] V. Pavlovic, M. Ciric, V. Jovanovic, and P. Stojanovic, "Platelet rich plasma: a short overview of certain bioactive components," Open Medicine, vol. 11, no. 1, pp. 242-247, 2016.

[56] A. J. Deucher, K. A. Simon, P. P. Vinha, and W. Fiori, "PRP as an antioxidant strategy," Free Radical Biology and Medicine, vol. 120, p. S119, 2018. 